Short communication

\title{
Nitric oxide mediates the amplification by interleukin- $1 \beta$ of neurogenic vasodilatation in the rat skin
}

\author{
Michael K. Herbert ${ }^{a, *}$, Peter Holzer ${ }^{\text {b }}$ \\ a Department of Anaesthesiology, University of Würzburg, Josef-Schneider-Strasse 2, D-97080 Würzburg, Germany \\ b Department of Experimental and Clinical Pharmacology, University of Graz, Universitätsplatz 4, A-8010 Graz, Austria
}

Received 6 April 1994; accepted 20 May 1994

\begin{abstract}
Blood flow in the plantar hindpaw skin of anaesthetized rats was measured by laser Doppler flowmetry. Intraplantar injection of interleukin- $1 \beta(50 \mathrm{pg})$ significantly enhanced the hyperaemic response to intraplantar capsaicin $(0.3 \mu \mathrm{g})$. Pretreatment with a neurotoxic dose of capsaicin reduced the capsaicin-evoked hyperaemia and prevented the facilitatory effect of interleukin- $1 \beta$. Blockade of nitric oxide formation by $N^{\mathrm{G}}$-nitro-L-arginine methyl ester failed to affect the capsaicin-evoked vasodilatation but abolished its amplification by interleukin-1 $\beta$. These data indicate that the enhancement by interleukin-1 $\beta$ of the capsaicin-induced hyperaemia involves thin afferent nerve fibres and depends on nitric oxide as essential intermediate.
\end{abstract}

Key words: Afferent nerve stimulation; Capsaicin; Cutaneous hyperemia; Interleukin-1 $\beta$; Neurogenic inflammation; Nitric oxide (NO)

\section{Introduction}

The cutaneous vasodilatation induced by topical administration of capsaicin is to a large extent mediated by activation of thin afferent nerve fibres and release of vasoactive peptides from their peripheral endings (Holzer, 1992). We have previously shown that the inflammatory cytokine interleukin- $1 \beta$ enhances the hyperaemia evoked by injection of capsaicin into the rat plantar hindpaw skin in a prostaglandin-dependent manner (Herbert and Holzer, 1994). Since the vasodilator effect of calcitonin gene-related peptide (CGRP), the major neurogenic mediator of the capsaicin-induced vasodilatation (Hughes and Brain, 1991; Brain et al., 1993), was not altered by interleukin-1 $\beta$ it was concluded that the effect of the cytokine was brought about by sensitization of afferent nerve endings to capsaicin (Herbert and Holzer, 1994).

This inference was further tested here by examining the effect of interleukin- $1 \beta$ in rats in which thin afferent neurones had been defunctionalized by systemic

\footnotetext{
* Corresponding author. Tel. [0043] (316) 380-4310; Fax [0043] (316) 380-4323.
}

pretreatment with a neurotoxic dose of capsaicin (Holzer, 1991). Another aim of this study was to explore a possible participation of nitric oxide (NO) in the interleukin- $\beta$-evoked augmentation of the vasodilator response to capsaicin. This possibility was envisaged from the findings that the effects of interleukin- $1 \beta$ on a variety of tissues and cells depend on the formation of $\mathrm{NO}$ as an intermediate messenger (Esplugues et al., 1993; Hogaboam et al., 1993; Inoue et al., 1993). Furthermore, it has been found that NO generated in the rat skin can give rise to afferent nerve-mediated vasodilatation (Holzer and Jocič, 1994).

\section{Materials and methods}

The experiments, approved by the Austrian Ministry of Science and Research, were carried out with female Sprague-Dawley rats (Versuchstierzuchtanstalt Himberg, Austria) weighing 180-245 g. The rats were anaesthetized with phenobarbitone ( $250 \mathrm{mg} \mathrm{kg}^{-1}$ i.p.), their trachea was cannulated, blood pressure monitored continuously via a catheter in the right carotid artery, and rectal temperature maintained at $37.5-38^{\circ} \mathrm{C}$ with a heating pad (Herbert and Holzer, 1994). Blood 
flow in the plantar skin of each hindpaw was recorded with a calibrated laser Doppler flowmeter (Periflux PF3, Perimed, Sweden) as described previously (Herbert and Holzer, 1994), blood flow being expressed in dimensionless perfusion units (PU).

The experimental procedure started after a $1 \mathrm{~h}$ equilibration period when interleukin- $1 \beta$ (50 pg in 10 $\mu 1$ fluid) was injected s.c. into the plantar side of the left paw while the right paw received vehicle $(10 \mu \mathrm{l}$ saline) only (time 0$)$. Forty min later capsaicin $(0.3 \mu \mathrm{g}$ in $10 \mu \mathrm{l}$ fluid) was injected into the same skin area (Herbert and Holzer, 1994). Cutaneous blood flow was measured at times 35, 45, 60 and 75 min (Herbert and Holzer, 1994). In study 1 this experimental protocol was tested on rats that had been pretreated with a neurotoxic dose of capsaicin $\left(125 \mathrm{mg} \mathrm{kg}^{-1}\right.$, s.c.) or its vehicle 2 weeks before the experiments. As described elsewhere (Holzer et al., 1992), the total dose of capsaicin was given in 4 injections over 2 days while the control rats received equal volumes of the vehicle. In study 2 the effects of $N^{\mathrm{G}}$-nitro-L-arginine methyl ester $\left(15 \mathrm{mg} \mathrm{kg}{ }^{-1}\right.$ given i.v. 30 min before the intraplantar injection of interleukin-1 $\beta$ ) and its enantiomer, $N^{\mathrm{G}}$. nitro-D-arginine methyl ester, were compared with that of the vehicle (saline, $1 \mathrm{ml} \mathrm{kg}^{-1}$ ).

Human recombinant interleukin-1 $\beta$ (Sigma, Deisenhofen, Germany), $N^{\mathrm{G}}$-nitro-L-arginine methyl ester and its enantiomer $N^{\mathrm{G}}$-nitro-D-arginine methyl ester (Bachem, Bubendorf, Switzerland) were dissolved in saline $(0.15 \mathrm{M} \mathrm{NaCl})$. Capsaicin (Serva, Heidelberg, Germany) was dissolved in absolute ethanol (30 mg $\mathrm{ml}^{-1}$ ) and diluted with saline such that the solution used for intraplantar injection contained $0.1 \%(\mathrm{w} / \mathrm{w})$ ethanol. The results are expressed as means \pm S.E.M. of $n$ rats. For statistical evaluation the PU values of the right and left paw, recorded 5 min after the capsaicin injection, were analyzed with the Wilcoxon matched-pairs signed rank test (two-tailed). Differences between different groups of rats were evaluated with the Mann-Whitney $U$-test. A value of $P<0.05$ was regarded as significant.

\section{Results}

Intraplantar injection of capsaicin to vehicle-pretreated rats increased cutaneous blood flow, a reaction that was significantly larger in the paw treated with interleukin- $\beta$ than in the paw treated with saline (Fig. 1). Pretreatment of rats with a neurotoxic dose of capsaicin did not significantly alter basal blood flow but inhibited the vasodilatation caused by acute intraplantar injection of capsaicin (Fig. 1). In the capsaicinpretreated rats, the hyperaemic response to intraplantar injection of capsaicin (increment of cutaneous blood flow by $32 \pm 6 \mathrm{PU}, n=6$ ) was not larger, but rather
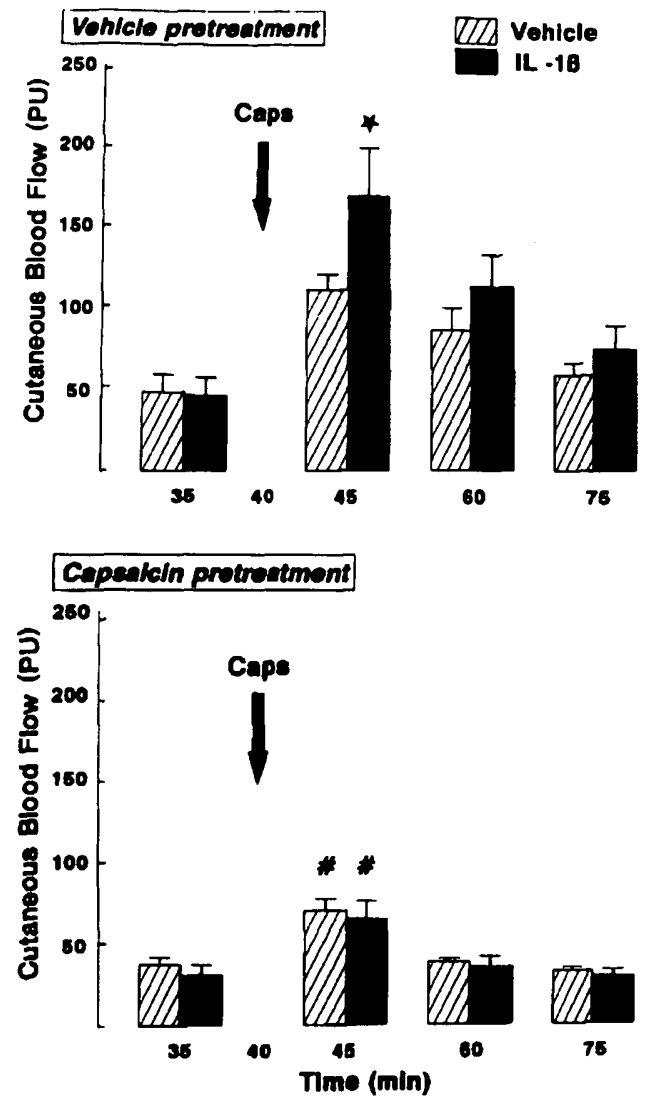

Fig. 1. Effect of interleukin-1 $\beta$ (IL-1 $\beta$ ) to enhance the cutaneous hyperaemia evoked by capsaicin (Caps) in vehicle- and capsaicin-pretreated rats. Capsaicin ( $125 \mathrm{mg} \mathrm{kg}^{-1}$ s.c.) or its vehicle were administered 2 weeks before the experiments. At time 0 of the experiment, vehicle ( $10 \mu \mathrm{l}$ saline) was injected into the plantar side of the right paw whilst the left paw was treated with $50 \mathrm{pg}$ IL-1 $\beta$. Forty min later Caps $(0.3 \mu \mathrm{g})$ was injected into each paw. Cutaneous blood flow in the right (hatched columns) and left (filled columns) hindpaw was recorded by laser Doppler flowmetry and expressed in perfusion units (PU). The bars represent means \pm S.E.M. of 6 rats. ${ }^{*} P<0.05$ versus respective values measured in the saline-treated paw (twotailed Wilcoxon matched-pairs signed rank test); ${ }^{*} P<0.05$ versus respective values measured in the vehicle-pretreated rats (two-tailed Mann-Whitney $U$-test).

smaller, than the response to intraplantar injection of saline (increment of cutaneous blood flow by $53 \pm 4$ PU, $n=6$ ). The effect of interleukin-1 $\beta$ to augment the capsaicin-induced vasodilatation was abolished by capsaicin pretreatment (Fig. 1). Mean arterial blood pressure in the capsaicin-pretreated rats $(87 \pm 7.5 \mathrm{~mm}$ $\mathrm{Hg}, n=6)$ did not significantly $(P=0.084)$ differ from that in the vehicle-pretreated rats $(102 \pm 15.7 \mathrm{~mm} \mathrm{Hg}$, $n=6$ ) as measured 5 min before the intraplantar injection of capsaicin.

$N^{G}$-nitro-L-arginine methyl ester, its enantiomer $N^{\mathrm{G}}$-nitro-D-arginine methyl ester and the vehicle, saline, were injected i.v. $30 \mathrm{~min}$ before the administration of interleukin-1 $\beta$. Neither $N^{\mathrm{G}}$-nitro-L-arginine methyl ester nor $N^{\mathrm{G}}$-nitro-D-arginine methyl ester al- 
tered basal blood flow in the plantar hindpaw skin to a significant extent when compared with the basal blood flow in saline-treated rats, although a trend towards a reduction was seen with $N^{\mathrm{G}}$-nitro-L-arginine methyl ester and a trend towards an increase was observed with $N^{\mathrm{G}}$-nitro-D-arginine methyl ester (Fig. 2). $N^{\mathrm{G}}$. nitro-L-arginine methyl ester and $N^{\mathrm{G}}$-nitro-D-arginine methyl ester also failed to alter the hyperaemic response to intraplantar injection of capsaicin in the paw treated with saline. $N^{\mathrm{G}}$-nitro-L-arginine methyl ester, however, prevented interleukin- $1 \beta$ from enhancing the capsaicin-induced vasodilatation whereas $N^{\mathrm{G}}$-nitro-Darginine methyl ester was without effect (Fig. 2). $N^{\mathrm{G}}$ nitro-L-arginine methyl ester led to an immediate rise of mean arterial blood pressure by $39 \pm 6 \mathrm{~mm} \mathrm{Hg}$ $(n=8)$ as measured $10 \mathrm{~min}$ post-injection, whereas mean arterial blood pressure rose by $11 \pm 5 \mathrm{~mm} \mathrm{Hg}$ $(n=8)$ only after the injection of $N^{G}$-nitro-D-arginine methyl ester and did not change after i.v. injection of saline $(-4 \pm 3 \mathrm{~mm} \mathrm{Hg}, n=8)$. $N^{\mathrm{G}}$-nitro-D-arginine methyl ester, however, led to a delayed increase in blood pressure. Thus, $5 \mathrm{~min}$ before the intraplantar injection of capsaicin (i.e., $65 \mathrm{~min}$ after the i.v. administration of $N^{\mathrm{G}}$-nitro-L-arginine methyl ester, $N^{\mathrm{G}}$-nitro$D$-arginine methyl ester or saline) mean arterial blood pressure in the saline-treated rats was $98 \pm 11 \mathrm{~mm} \mathrm{Hg}$ $(n=8)$ compared with $113 \pm 25 \mathrm{~mm} \mathrm{Hg}(n=8$, not significantly different versus saline) in rats injected with $N^{\mathrm{G}}$-nitro-L-arginine methyl ester and $129 \pm 26$ mm $\mathrm{Hg}(n=8 ; P<0.01$ versus saline, not significantly different versus $N^{\mathrm{G}}$-nitro-L-arginine methyl ester) in rats injected with $N^{\mathrm{G}}$-nitro-D-arginine methyl ester.

\section{Discussion}

The present data confirm that interleukin- $1 \beta$ enhances the capsaicin-induced increase of blood flow in the plantar skin of the rat hindpaw (Herbert and Holzer, 1994). Pretreatment of rats with a neurotoxic dose of capsaicin (Holzer, 1991) showed that the vasodilator response to capsaicin requires an intact innervation of the skin by thin afferent neurones. The residual vasodilatation seen in capsaicin-pretreated rats is likely to be a reaction to the injection per se, since it was not higher than the vasodilatation caused by intraplantar injection of saline alone. These findings are consistent with the notion that the capsaicin-induced rise of cutaneous blood flow is mediated by stimulation of fine afferent nerve fibres (Holzer, 1992) and release of the vasodilator transmitter CGRP (Hughes and Brain, 1991; Brain et al., 1993). Hence, the absence of any effect of interleukin- $\beta$ in capsaicin-pretreated rats implies that the cytokine augments the neurogenic component in the vasodilator response to capsaicin by sensitizing afferent nerve fibres to noxious stimuli. This
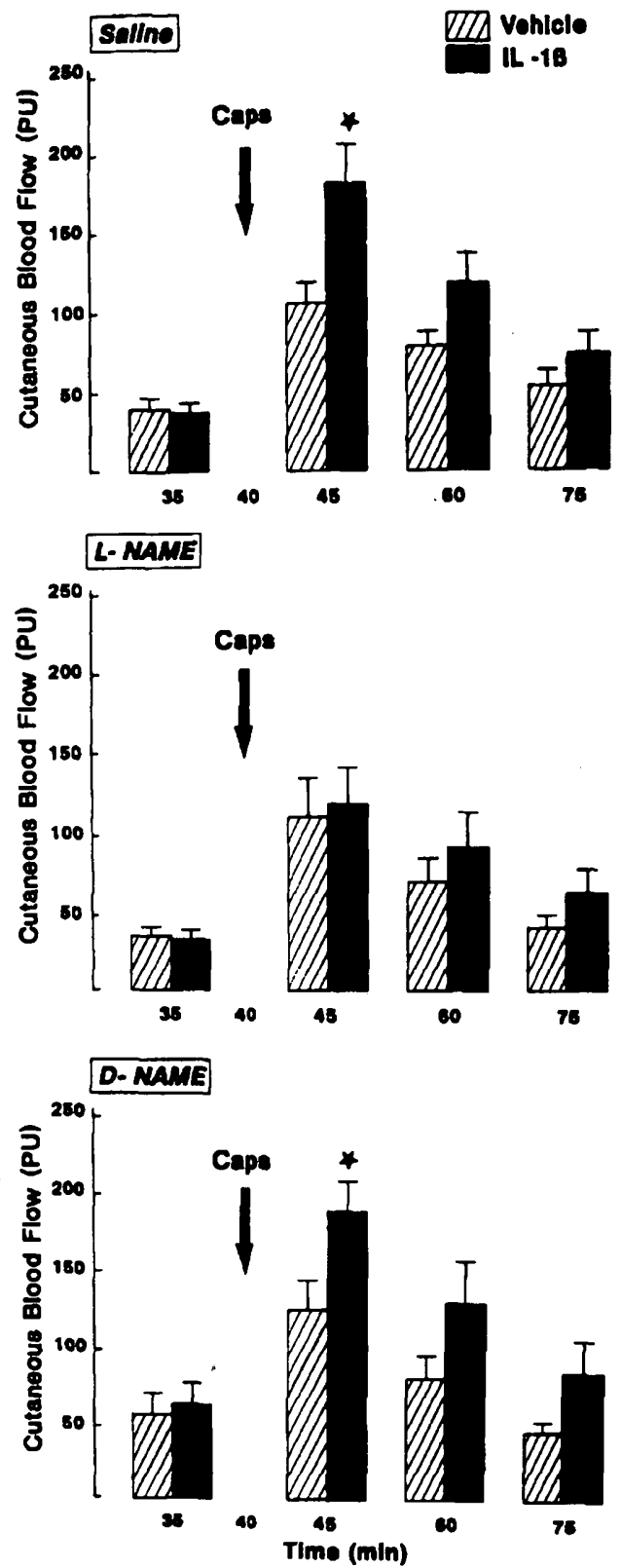

Fig. 2. Effect of interleukin-1 $\beta$ (IL-1 $\beta$ ) to enhance the cutaneous hyperaemia evoked by capsaicin (Caps) in rats treated with saline, $N^{\mathrm{G}}$-nitro-L-arginine methyl ester (L-NAME) or $N^{\mathrm{G}}$-nitro-D-arginine methyl ester (D-NAME). Saline $\left(1 \mathrm{ml} \mathrm{kg}^{-1}\right), \mathrm{L}-\mathrm{NAME}$ and D-NAME (each $15 \mathrm{mg} \mathrm{kg}^{-1}$ ) were injected i.v. $30 \mathrm{~min}$ before the administration of IL-1 $\beta$. At time 0 of the experiment, vehicle ( $10 \mu \mathrm{l}$ saline) was injected into the plantar side of the right paw whilst the left paw was treated with $50 \mathrm{pg}$ IL-1 $\beta$. Forty min later Caps $(0.3 \mu \mathrm{g})$ was injected into each paw. Cutaneous blood flow in the right (hatched columns) and left (filled columns) hindpaw was recorded by laser Doppler flowmetry and expressed in perfusion units (PU). The bars represent means \pm S.E.M. of 8 rats. ${ }^{*} P<0.05$ versus respective values measured in the saline-treated paw (two-tailed Wilcoxon matched-pairs signed rank test).

inference is further supported by the inability of interleukin- $1 \beta$ to enhance the vasodilator response to CGRP, which rules out the possibility that the cytokine 
acts by increasing the sensitivity of blood vessels to vasodilator mediators (Herbert and Holzer, 1994).

Important for the understanding as to how interleukin- $1 \beta$ sensitizes afferent nerve fibres is the finding that the cytokine-induced augmentation of the hyperaemic response to capsaicin depended on the formation of $\mathrm{NO}$ as an intermediate messenger. The involvement of NO was demonstrated with the NO synthase inhibitor $N^{\mathrm{G}}$-nitro-L-arginine methyl ester (Gardiner et al., 1990; Rees et al., 1990) which prevented the sensitizing action of interleukin- $\beta$ in an enantiomer-specific manner. $N^{\mathrm{G}}$-nitro-D-arginine methyl ester, which is said to be inactive on the NO synthase (Gardiner et al., 1990; Rees et al., 1990), did not interfere with the action of interleukin- $1 \beta$ but led to a delayed increase in blood pressure that did not differ from the prompt rise of blood pressure caused by $N^{\mathrm{G}}$-nitro-L-arginine methyl ester. It follows that the hypertension caused by $N^{G}$-nitro-L-arginine methyl ester cannot be held responsible for the concomitant suppression of the ability of interleukin-1 $\beta$ to facilitate the vasodilator response to capsaicin. The activity of $N^{\mathrm{G}}$-nitro-D-arginine methyl ester to elevate blood pressure, which has been noted previously (Holzer et al., 1993), is not understood at present. $N^{\mathrm{G}}$-nitro-L-arginine methyl ester was given systemically since this route of administration did not significantly diminish cutaneous blood flow, whereas local application of the drug has been observed to reduce skin blood flow to a significant extent (Holzer and Jocič, 1994). The use of S.c. $N^{\mathrm{G}}$-nitro-L-arginine methyl ester was regarded as impractible, therefore, given that local $N^{\mathrm{G}}$-nitro-L-arginine methyl ester could inhibit vasodilator responses solely by virtue of its vasoconstrictor effect. Such a functional antagonism might be responsible for the inhibitory effect of topical $N^{\mathrm{G}}$-nitro-L-arginine methyl ester on capsaicin-induced vasodilatation (Brain et al., 1993), an effect that was not observed after i.v. administration of the drug. The effectiveness of $N^{\mathrm{G}}$-nitro-L-arginine methyl ester (15 $\mathrm{mg} \mathrm{kg} \mathrm{kg}^{-1}$ i.v.) as a blocker of NO synthase has been established in a previous study (Holzer et al., 1993).

The participation of NO in the action of interleukin$1 \beta$ to augment the capsaicin-evoked rise of cutaneous blood flow needs to be seen in context with the reported involvement of cyclooxygenase products such as prostaglandins in this action of the cytokine (Herbert and Holzer, 1994). The question arises as to cellular sources and the sequential mechanism of action of NO and prostaglandins. As both autacoids are shortlived biological messengers it has to be assumed that NO and cyclooxygenase products are generated by interleukin- $1 \beta$ in close vicinity of, or even within, afferent nerve endings so that they can contribute to the sensitization process. Two cellular systems may play a particularly important role in the action of interleukin- $1 \beta$ to augment the hyperaemic response to capsaicin. Firstly,
NO is produced by rat mast cells in response to interleukin-1 $\beta$ (Hogaboam et al., 1993) within a time frame of $30 \mathrm{~min}$ and influences the release of other inflammatory mediators (Hogaboam et al., 1993). As mast cells are often juxtaposed to blood vessels and afferent nerve endings (Holzer, 1992) it could be hypothesized that interleukin- $\beta$ acts on these cells to stimulate the sequential formation of NO and prostaglandins which, in turn, contribute to the sensitization of afferent nerve endings. In addition, NO generated in the rat skin can by itself give rise to afferent nerve-mediated vasodilatation (Holzer and Jocič, 1994). Secondly, interleukin$1 \beta$ has been shown to stimulate the consecutive formation of $\mathrm{NO}$ and prostaglandin $\mathrm{E}_{2}$ in vascular smooth muscle cells (Inoue et al., 1993). It is not clear, however, whether this effect of the cytokine has a bearing on the current findings because the cytokine's effect on vascular smooth muscle cells depends on the induction of NO synthase and requires several hours to develop (Inoue et al., 1993).

In conclusion, interleukin- $\beta$ has been shown to augment the cutaneous hyperaemic response to capsaicin by a sensitizing action on afferent nerve fibres, an action which involves NO. These findings emphasize a close interaction between immune mediators (interleukin-1 $\beta$ ), intermediate messengers (NO, prostaglandins) and neurogenic factors (CGRP) in the control of vascular functions and inflammatory processes in the skin.

\section{Acknowledgements}

The authors are grateful to Dr. R. Amann for his comments on the manuscript and to Mr. W. Schluet and Ms. M. Jocič for providing organizational assistance. The study was supported by the Foundation for the Advancement of Research in Anaesthesia and Analgesia (e. V., Würzburg), the Austrian Science Foundation (grant P9473MED) and the Dr. Kolassa Foundation for Rheumatological Research (Austria).

\section{References}

Brain, S.D., S.R. Hughes, H. Cambridge and G. O'Driscoll, 1993, The contribution of calcitonin gene-related peptide (CGRP) to neurogenic vasodilator responses, Agents Actions 38, C19.

Esplugues, J.V., M.D. Barrachina, S. Calatayud, J.M. Pique and B.J.R. Whittle, 1993, Nitric oxide mediates the inhibition by interleukin-1 $\beta$ of pentagastrin-stimulated rat gastric acid secretion, Br. J. Pharmacol. 108, 9.

Gardiner, S.M., A.M. Compton, P.A. Kemp and T. Bennett, 1990 , Regional and cardiac haemodynamic effects of $N^{\mathrm{G}}$-nitro-Larginine methyl ester in conscious, Long Evans rats, Br. J. Pharmacol. 101, 625 .

Herbert, M.K. and P. Holzer, 1994, Interleukin-1 $\beta$ enhances capsaicin-induced neurogenic vasodilatation in the rat skin, $\mathrm{Br}$. $\mathrm{J}$. Pharmacol. 111, 681.

Hogaboam, C.M., A.D. Befus and J.L. Wallace, 1993, Modulation of rat mast cell reactivity by IL-1 $\beta$. Divergent effects on nitric oxide and platelet-activating factor release, J. Immunol. 151, 3767. 
Holzer, P., 1991, Capsaicin: cellular targets, mechanisms of action, and selectivity for thin sensory neurons, Pharmacol. Rev. 43, 143.

Holzer, P., 1992, Peptidergic sensory neurons in the control of vascular functions: mechanisms and significance in the cutaneous and splanchnic vascular beds, Rev. Physiol. Biochem. Pharmacol. $121,49$.

Holzer, P. and M. Jocič, 1994, Nitric oxide-induced neurogenic vasodilatation in the rat skin, $\mathrm{Br}$. J. Pharmacol. in press.

Holzer, P., I.T. Lippe and R. Amann, 1992, Participation of capsaicin-sensitive afferent neurons in gastric motor inhibition caused by laparotomy and intraperitoneal acid, Neuroscience 48,715 .

Holzer, P., I.T. Lippe, M. Jocič, C. Wachter, R. Erb and A. Heinemann, 1993, Nitric oxide-dependent and -independent hyperaemia due to calcitonin gene-related peptide in the rat stomach, Br. J. Pharmacol. 110, 404.
Hughes, S.R. and S.D. Brain, 1991, A calcitonin gene-related peptide (CGRP) antagonist $\left(\mathrm{CGRP}_{\mathbf{8 - 3 7}}\right)$ inhibits microvascular responses induced by CGRP and capsaicin in skin, Br. J. Pharmacol. 104, 738.

Inoue, T., K. Fukuo, S. Morimoto, E. Koh and T. Ogihara, 1993, Nitric oxide mediates interleukin-1-induced prostaglandin $E_{2}$ production by vascular smooth muscle cells, Biochem. Biophys. Res. Commun. 194, 420.

Rees, D.D., R.M.J. Palmer, R. Schulz, H.F. Hodson and S. Moncada, 1990, Characterization of three inhibitors of endothelial nitric oxide synthase in vitro and in vivo, Br. J. Pharmacol. 101, 746. 